\title{
A Study of the Intermetallic Compound Growth in Flip-Chip Packages under Thermal Loading
}

\author{
C. Xu ${ }^{1,2}$, Z.W. Zhong ${ }^{1}$, W.K. Choi ${ }^{2}$ \\ 1. School of Mechanical and Aerospace Engineering, Nanyang Technological University, Singapore \\ 2. STATS ChipPAC Pte Ltd, Singapore \\ E-mail:xuch0005@e.ntu.edu.sg,mzwzhong@ntu.edu.sg,wonkyoung.choi@Statschippac.com
}

Received: 22 September 2018; Accepted: 3 October 2018; Available online: 15 February 2019

\begin{abstract}
The intermetallic compound layers in solder bumps have the brittle feature and can easily fracture under thermal or mechanical loading. Therefore, the intermetallic compound is an issue for the fracture reliability of the solder bumps. In this work, the intermetallic compound growth before and after high temperature storage tests was investigated. The experiment results revealed that the solder bumps with nickel layers could reduce the intermetallic compound growth rate. The nickel layer, which was added in between $\mathrm{Cu}$ and $\mathrm{SnAg}$ for top solder bumps, was an important factor controlling the intermetallic compound thickness. It was hard to tell the intermetallic compound thickness at time zero; at the time of 147 hours, the intermetallic compound grew to 3.25 $\mu \mathrm{m}$; at the time of 294 hours, the intermetallic compound grew to $5.25 \mu \mathrm{m}$. However, the solder joints were still in good condition.
\end{abstract}

Keywords: Intermetallic compound; Thermal loading; Fracture reliability; Solder joint.

\section{Introduction}

Nowadays the semiconductor chips demand smaller packages and higher functionality, which requires the chips to have more inputs/outputs. The bump height and pitch must be reduced in order to fulfill this requirement. The initial wafer-level packaging is known as the wafer-level chip-scale packaging technology [1]. The fan-out waferlevel package technology [2] is a further development of the wafer-level chip-scale packaging technology [3, 4], and its most significant aspect is the fan-out area [5,6]. The fan-out wafer-level package input/output numbers are much higher than the wafer-level chip-scale packaging input/output numbers. The fan-out wafer-level packages $[7,8]$ can be further developed to $2.5 \mathrm{D} / 3 \mathrm{D}$ fan-out wafer-level packages $[9,10]$ or fan-out package-on-package $[11,12]$ through laser ablation or through silicon via (TSV) technology.

The silicon die must be thin so as to build a thin over-molded [13] structure fan-out wafer-level package [14]. The fan-out wafer-level package also shows better thermal-mechanical reliability performance $[15,16]$ than other packages such as plastic ball grid array packages [17]. The next generation wafer level packaging turns to panel carrier [18], and thus the yield will increase exponentially and the cost will reduce significantly [19]. The threepoint bending test [20] is the most popular method for the evaluation of silicon strength. Some research has shown that the silicon own profile factors do not affect the silicon strength obviously and directly [21, 22]. There are three widely accepted effect factors of silicon strength: silicon surface defects, silicon edge defects and weak planes of silicon crystal lattice [23].

Compared with other flip chip technologies $[24,25]$, three-dimensional chip stacking packaging with micro bumps has many advantages: better electrical performance, shorter interconnect lengths, thinner package profile, and enhanced function density. Zhan et al. [26] proposed a high-density three-dimensional chip-on-chip packaging technology with lead-free solder micro-bump interconnections. In their work, they used underfill dispensing and thermal compression bonding processes and assembled a number of $\mathrm{Cu} / \mathrm{Ni}$ micro-bumps on an interposer or silicon chip with $\mathrm{Sn} 2.5 \mathrm{Ag}$ solders.

However, many issues remain to be addressed before the technology can be feasible. The shorter bump height and fine pitch can benefit the atomic diffusion, but the intermetallic compound layer in the solder joint has a serious effect on the solder joint fracture reliability and thus the whole package reliability. The intermetallic compound is very brittle and may fracture under certain thermal or mechanical loading. Therefore, a thick intermetallic compound layer is a potential problem to the package reliability. A foreign material layer may prevent the copper from its reaction with tin [27]. A thin intermetallic compound layer of a few micrometres thick could create a good metallurgical bonding strength [28]. The foreign material layer might prevent the intermetallic compound growth [29-31]. 
One research [32] found that the solder bump standoff height was a factor that could affect the solder joint reliability. The researchers conducted the research through mathematics prediction calculation, experiment and FEM etc. The research was very detailed, but the conclusions stated that the solder bump standoff height was not critical to the intermetallic compound formation. This point was verified by a solder bump material study [33]. $\mathrm{Cu}$ and $\mathrm{Ni}$ atoms could cross hundreds of micrometres in a few hundred hours to interact with other atoms even in the solid state. The solder bump topology was an important factor, which affected the intermetallic compound formation. This was because the thermal-mechanical stress affected the fine solder bump pitch package reliability during thermal testing [34]. But, the research only showed a single solder bump pitch. There were not any comparisons between different solder bump pitches within the same package.

Another research [35] showed the function of a Ni layer for $100-\mu \mathrm{m}$ and $200-\mu \mathrm{m}$ micro bump pitches could prevent the intermetallic compound growth effectively. But, the bump pitches were not fine pitches. The solder bump topology might accelerate the intermetallic compound formation. However, the solder bump material was the source of intermetallic compound layers, and resulted in the intermetallic compound layer formation difficulty. The tin/silver/copper (SAC) solder alloy is the most popular selection of packaging manufacturers. The Ag content in SAC solder bumps affects the Ag3Sn intermetallic compound formation [36, 37]. According to atom chemistry properties, the reaction between some atoms requires higher energy. Heat is the source of energy. Therefore, the intermetallic compound layer formation always occurs in heat-related processes, like the reflow process and thermal reliability tests etc. If the external heat is not enough to break the existing chemical bond, then the new composition formation is stopped. Vice versa if the chemical bond threshold is low, then the new composition formation does not require much energy. Based on this property, the intermetallic compound growth rate may be reduced significantly through adding a foreign material in the solder bumps, like antimony (Sb) [38, 39]. Nickel (Ni) could be another foreign material to reduce the intermetallic compound growth rate [40]. However, there was not any concentrated and detailed research to show this. The shorter bump height and fine pitch can benefit the atomic diffusion. The intermetallic compound is very brittle and may fracture under certain thermal or mechanical loading. Therefore, a thick intermetallic compound layer is a potential problem to the package reliability.

In this work, the intermetallic compound growth before and after high temperature storage tests was investigated, so as to study the fracture reliability of the solder bumps with nickel layers added.

\section{Methodology}

The samples were $14 \mathrm{~mm} \times 20 \mathrm{~mm}$ flip chip packages in this experiment. There were two dies with different sizes in each package. The big die had a solder bump pitch of $80 \mu \mathrm{m}$; the smaller die had solder bump pitches of $40 \mu \mathrm{m}$ and $60 \mu \mathrm{m}$. The solder bump diameter was $20 \mu \mathrm{m}$. There were two batches of samples, and they were named S1 and S2 samples. Table 1 lists the solder bump composition of these samples. The materials of the top solder bumps were $\mathrm{Cu} / \mathrm{SnAg}$ and $\mathrm{Cu} / \mathrm{Ni} / \mathrm{SnAg}$. The material of the interposer side solder bumps was $\mathrm{Cu} / \mathrm{Ni} / \mathrm{SnAg}$.

The top die was attached by using a chip attach machine with a bonding force of $4 \mathrm{~kg}$ and bonding time of 1 second. The whole wafer suffered a reflow process after the chip-attach process. An underfill process was applied and the underfill was cured at $180{ }^{\circ} \mathrm{C}$ for 4 hours.

Table 1. Solder bump composition of samples.

\begin{tabular}{ccccc}
\hline & \multicolumn{2}{c}{ Die side } & \multicolumn{2}{c}{ Interposer side } \\
\cline { 2 - 5 } & Composition & Thickness $(\mu \mathrm{m})$ & Composition & Thickness $(\mu \mathrm{m})$ \\
\hline S1 & $\mathrm{Cu} / \mathrm{SnAg}$ & $25 / 15$ & $\mathrm{Cu} / \mathrm{Ni} / \mathrm{SnAg}$ & $3 / 3 / 5$ \\
$\mathrm{~S} 2$ & $\mathrm{Cu} / \mathrm{Ni} / \mathrm{SnAg}$ & $25 / 3 / 15$ & $\mathrm{Cu} / \mathrm{Ni} / \mathrm{SnAg}$ & $3 / 3 / 5$ \\
\hline
\end{tabular}

The conditions of the accelerated high temperature storage test were almost the same as that of the normal high temperature storage test, but the tested devices were stored at a higher temperature. The temperature of the accelerated high temperature storage test was $175{ }^{\circ} \mathrm{C}$, which was higher than $150{ }^{\circ} \mathrm{C}$ for the normal high temperature storage test. There were 50 confirming units (ten each from top, left, center, right and bottom) selected from each wafer for the high temperature storage test. The readout points were 147 hours and 294 hours. Two samples were taken out at each readout point, and cross sectioning was performed for the samples with 40, 60 and $80 \mu \mathrm{m}$ solder joint pitches. We also selected some time-zero samples for comparison purposes. The samples were polished without the cold mount. A scanning electron microscope was utilised to observe the microstructure of the intermetallic compound and make the measurement.

\section{Experiment results}


Figure 1 shows an image of a solder joint condition of an S1 sample at time zero. There is a clear thickness difference of the intermetallic compound layers on the two sides of the bump. The die (top) side intermetallic compound layer thickness is about $3 \mu \mathrm{m}$, but the interposer-side intermetallic compound layer is difficult to observe. The die-side copper column is etched by the intermetallic compound layer and its shape is changed. However, the copper and nickel layers still keep their shapes on the interposer-side of the solder.

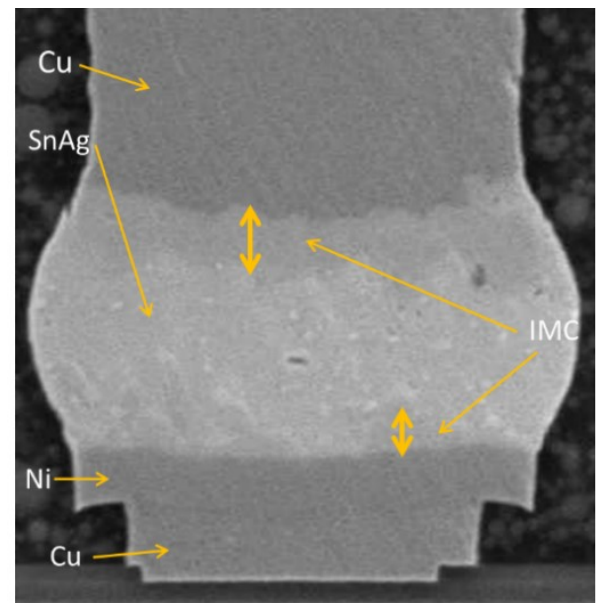

Figure 1. A solder joint of an S1 sample at time zero without a nickel layer on the top-side.

Figure 2 shows the cross-section images of sample-S1 solder joints after the 147 hours and 294 hours accelerated high temperature storage test. After the 147 hours accelerated high temperature storage test, the die (top) side intermetallic compound layers almost grew twice compared with time-zero samples. However, the interposer-side intermetallic compound layers grew only a little from 'difficult to observe' to 'can be observed'. For the $80-\mu \mathrm{m}$ pitch, the intermetallic compound growth rate was the fastest among the three pitches, and the top and bottom intermetallic compound layers almost joined together. The die-side intermetallic compound layers etched the copper column continuously, and the copper column shape became trapezia from rectangles. The copper column thickness was also reduced. This means that more $\mathrm{Cu}$ atoms participated in the intermetallic compound formation.
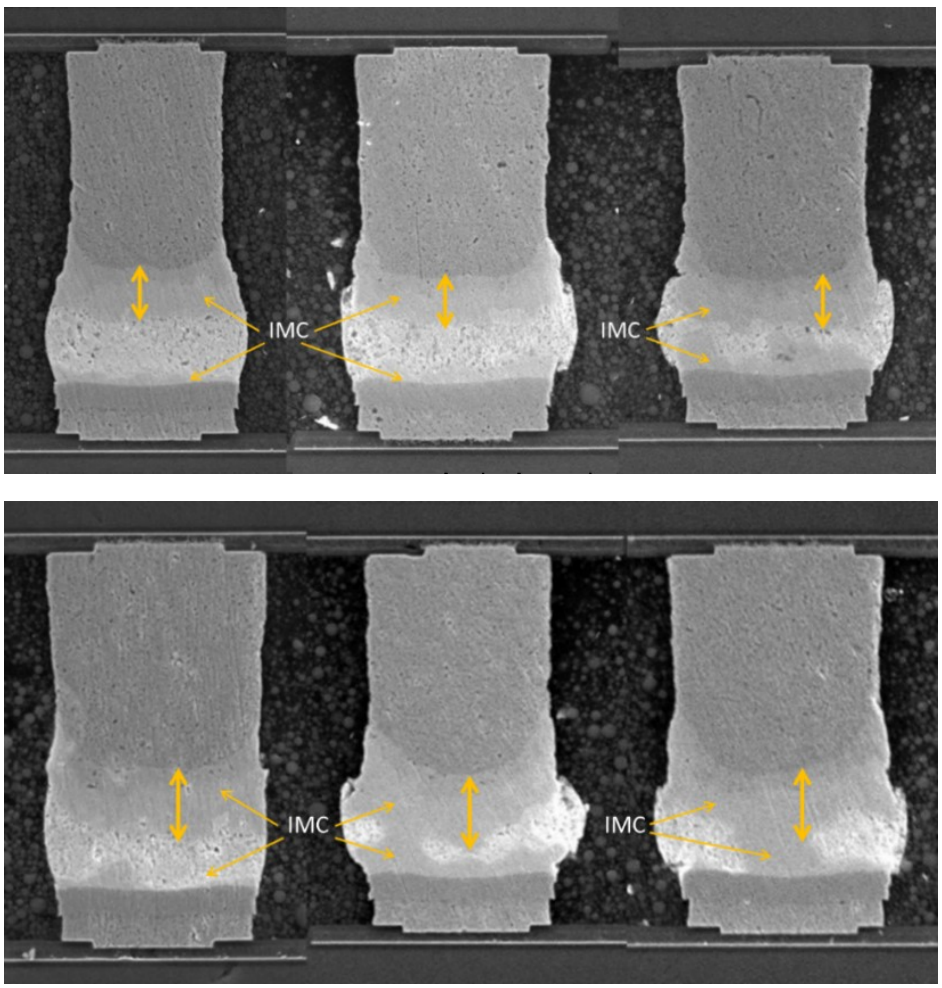

Figure 2. The cross-section images of sample-S1 solder joints with 40, 60 and $80 \mu \mathrm{m}$ pitches after 147 hours (top) and 294 hours (bottom) accelerated high temperature storage tests. 
After the 294 hours accelerated high temperature storage test, the die (top) side intermetallic compound layers grew continuously and rapidly. They took up the dominant solder joint space. For the 60 and $80 \mu \mathrm{m}$ pitches, their top and bottom intermetallic compound layers joined together and became integrated. For the $40 \mu \mathrm{m}$ pitches, their top and bottom intermetallic compound layers almost joined together. Anyway, the top side intermetallic compound layers were the major contributor. The die-side intermetallic compound layers etched the copper column continuously, and the copper column shape became rounds from trapezia. The copper column thickness was also reduced further. For the interposer-side solder joints, their $\mathrm{Cu}$ and $\mathrm{Ni}$ layers, especially the $\mathrm{Cu}$ layers, still retained their shapes.

According to the above observations, Ni has an obvious effect on the intermetallic compound growth. The die side intermetallic compound layers (without Ni) always grow rapidly and take up the major solder joint space. However, the interposer side intermetallic compound layers (with Ni) grow slowly, and they merge with die side intermetallic compound layers passively.

According to the above experiment results, the intermetallic compound growth rate on the interposer side was much slower than that on the die side. This might be because of the nickel layer of the interposer-side solder bump. Therefore, a 3- $\mu \mathrm{m}$ nickel layer was added in the die-side solder bumps of S2 samples. We expected that this nickel layer could reduce the die-side intermetallic compound growth.

Figure 3 gives the cross-section images of the solder joints before the reliability test for three different pitches of S2 samples. There is a nickel layer added in the die (top) side solder bump, and hence the composition of the die-side solder bump is $\mathrm{Cu} / \mathrm{Ni} / \mathrm{SnAg}$. The nickel layer can prevent the $\mathrm{SnAg}$ compound from climbing up and reduce the chance of $\mathrm{Cu}, \mathrm{Sn}$ and $\mathrm{Ag}$ compound formation.

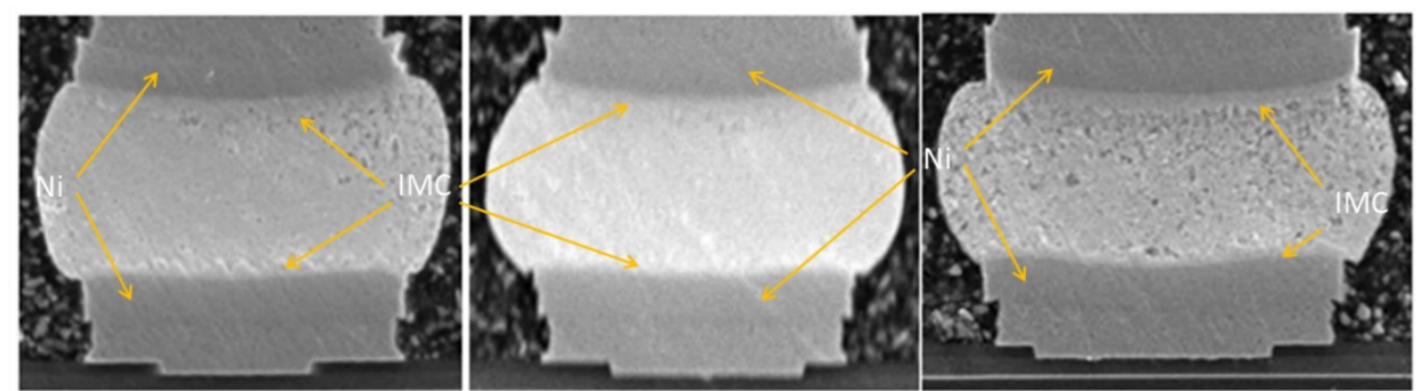

Figure 3. The cross-section images of solder joints with 40, 60 and $80 \mu \mathrm{m}$ pitches (with nickel layers on both sides) at time zero.

Figure 4 shows the images of the solder joints after 147 hours and 294 hours accelerated high temperature storage tests. The intermetallic compound layers grow up continuously throughout the tests; however, the test result is much better than that shown in Figure 2. No any intermetallic compounds join together after the 294 hours accelerated high temperature storage test. No any intermetallic compounds appear in the copper column of topside solder bumps after the 147 hours accelerated high temperature storage test, and the nickel layer prevents the intermetallic compound growth successfully at this time point. The thickness of intermetallic compound layer is $3.25 \mu \mathrm{m}$ after the 147 hours accelerated high temperature storage test, and $5.25 \mu \mathrm{m}$ after the 294 hours accelerated high temperature storage test. The intermetallic compound layers grew $2 \mu \mathrm{m}$ after 147 hours of accelerated high temperature storage testing. However, the solder joints were in good condition, and they were still functional.

\section{Conclusions}

This experimental study revealed that the nickel layer, which was added in between $\mathrm{Cu}$ and $\mathrm{SnAg}$ for top-side solder bumps, was an important factor controlling the intermetallic compound thickness. It has proved that the nickel layer has an effective impact on the intermetallic compound thickness. We applied accelerated high temperature storage tests to the samples for 147 and 294 hours respectively at $175{ }^{\circ} \mathrm{C}$. It was hard to tell the intermetallic compound thickness at time zero; at the time of 147 hours, the intermetallic compounds grew to 3.25 $\mu \mathrm{m}$; at the time of 294 hours, the intermetallic compound grew to $5.25 \mu \mathrm{m}$. However, the solder joints were still in good condition. Moreover, the intermetallic compound growth rate remained the same regardless the solder bump composite. 

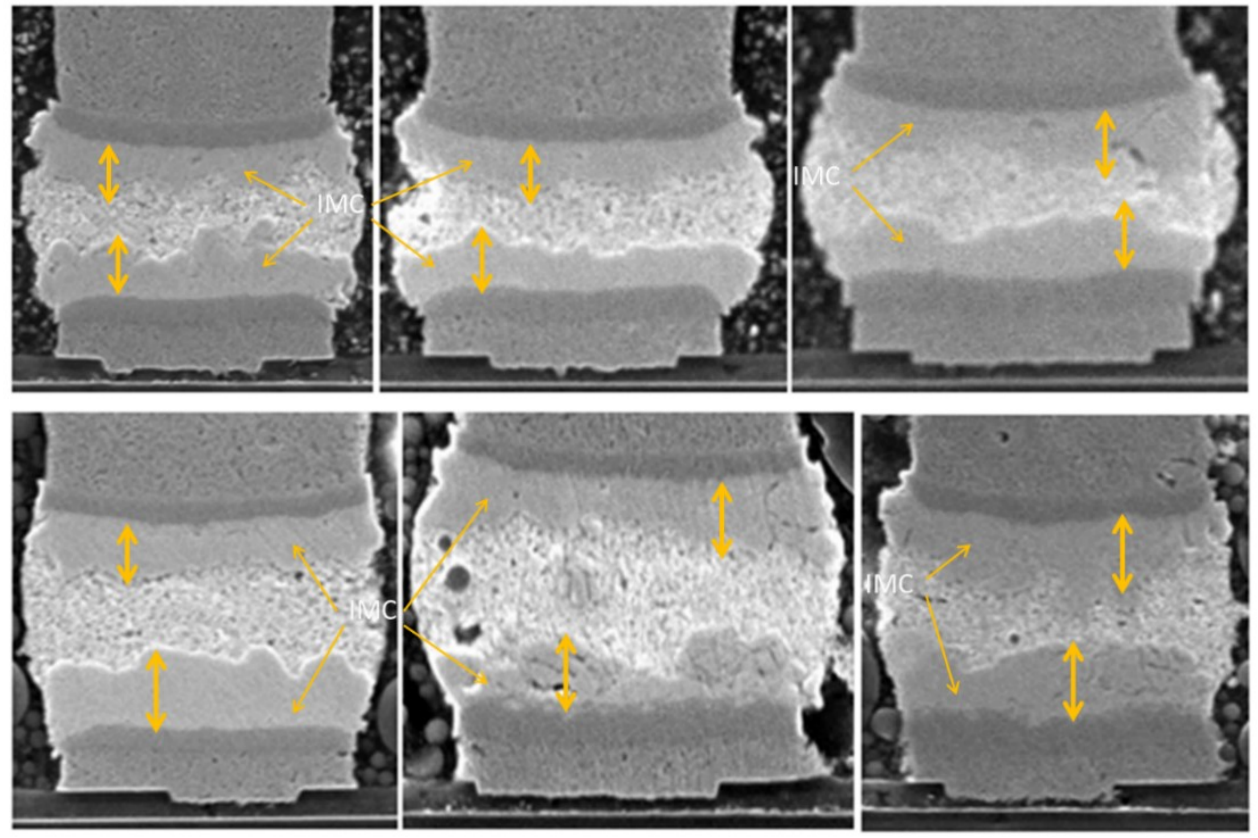

Figure 4. The cross-section images of sample-S2 solder joints with 40, 60 and $80 \mu \mathrm{m}$ pitches (with nickel layers on both sides) after 147 hours (top) and 294 hours (bottom) accelerated high temperature storage tests.

\section{Acknowledgments}

The financial support of this work is provided by the Economic Development Board Singapore Industrial PostGraduate Programme research grant. The authors are grateful to the support from the Economic Development Board Singapore and STATS ChipPAC Pte Ltd.

\section{References}

[1] M. Brunnbauer, E. Furgut, G. Beer, and T. Meyer. Embedded wafer level ball grid array (eWLB). Presented at the Electronics Packaging Technology Conference, 2006. EPTC '06. 8th, 2006.

[2] C. Xu, Z. W. Zhong, and W. K. Choi. Numerical and Experimental Study of Fan-Out Wafer Level Package Strength. In 2017 IEEE 67th Electronic Components and Technology Conference (ECTC), 2017. p. 21872192.

[3] Jin Yonggang, X. Baraton, S. W. Yoon, Lin Yaojian, P. C. Marimuthu, V. P. Ganesh, et al. Next generation eWLB (embedded wafer level BGA) packaging. Presented at the Electronics Packaging Technology Conference (EPTC), 2010 12th, 2010.

[4] S. W. Yoon, Lin Yaojian, S. Gaurav, Jin Yonggang, V. P. Ganesh, T. Meyer, et al. Mechanical characterization of next generation eWLB (embedded wafer level BGA) packaging. Presented at the Electronic Components and Technology Conference (ECTC), 2011 IEEE 61st, 2011.

[5] Yoon Seung Wook, Lin Yaojian, and P. C. Marimuthu. Development and characterization of 300 $\mathrm{mm}$ large panel eWLB (embedded wafer level BGA). Presented at the Microelectronics and Packaging Conference (EMPC), 2011 18th European, 2011.

[6] M. Prashant, Yoon Seung Wook, Lin Yaojian, and P. C. Marimuthu. Cost effective 300mm large scale eWLB (embedded Wafer Level BGA) technology. Presented at the Electronics Packaging Technology Conference (EPTC), 2011 IEEE 13th, 2011.

[7] K. Pressel, G. Beer, T. Meyer, M. Wojnowski, M. Fink, G. Ofner, et al. Embedded wafer level ball grid array (eWLB) technology for system integration. Presented at the CPMT Symposium Japan, 2010 IEEE, 2010.

[8] Jin Yonggang, J. Teysseyrex, X. Baraton, S. W. Yoon, Lin Yaojian, and P. C. Marimuthu. Advanced packaging solutions of next generation eWLB technology. Presented at the Electronics Packaging Technology Conference (EPTC), 2011 IEEE 13th, 2011.

[9] M. Wojnowski and K. Pressel. Embedded wafer level ball grid array (eWLB) technology for high-frequency system-in-package applications. Presented at the Microwave Symposium Digest (IMS), 2013 IEEE MTT-S International, 2013. 
[10] Yoon Seung Wook, J. A. Caparas, Lin Yaojian, and P. C. Marimuthu. Advanced low profile PoP solution with embedded wafer level PoP (eWLB-PoP) technology. Presented at the Electronic Components and Technology Conference (ECTC), 2012 IEEE 62nd, 2012.

[11] Jin Yonggang, J. Teysseyre, X. Baraton, S. W. Yoon, Lin Yaojian, and P. C. Marimuthu. Development and characterization of next generation eWLB (embedded Wafer Level BGA) packaging. Presented at the Electronic Components and Technology Conference (ECTC), 2012 IEEE 62nd, 2012.

[12] Yoon Seung Wook, P. Tang, R. Emigh, Lin Yaojian, P. C. Marimuthu, and R. Pendse. Fanout flipchip eWLB (embedded Wafer Level Ball Grid Array) technology as 2.5D packaging solutions. Presented at the Electronic Components and Technology Conference (ECTC), 2013 IEEE 63rd, 2013.

[13] C. Xu, Z. W. Zhong, and W. K. Choi. Epoxy molding compound effect on fan-out wafer level package strength during post-mold thermal process. In 2017 16th IEEE Intersociety Conference on Thermal and Thermomechanical Phenomena in Electronic Systems (ITherm), 2017. p. 1388-1392.

[14] C. Xu, Z. W. Zhong, and W. K. Choi. Thermal test effect on fan-out wafer level package strength. In 2017 12th International Microsystems, Packaging, Assembly and Circuits Technology Conference (IMPACT), 2017. p. 271-274.

[15] C. Xu, Z. W. Zhong, and W. K. Choi. Effect of high temperature storage on fan-out wafer level package strength. In 2017 China Semiconductor Technology International Conference (CSTIC), 2017. p. 1-3.

[16] C. Xu, Z. W. Zhong, and W. K. Choi. Thermal effect on fan-out wafer level package strength. In 2016 IEEE 18th Electronics Packaging Technology Conference (EPTC), 2016. p. 700-703.

[17] C. C. Liu, S. M. Chen, F. W. Kuo, H. N. Chen, E. H. Yeh, C. C. Hsieh, et al. High-performance integrated fan-out wafer level packaging (InFO-WLP): Technology and system integration. Presented at the 2012 IEEE International Electron Devices Meeting (IEDM), 2012.

[18] T. Braun, S. Raatz, S. Voges, R. Kahle, V. Bader, J. Bauer, et al. Large area compression molding for Fanout Panel Level Packing. Presented at the 2015 IEEE 65th Electronic Components and Technology Conference (ECTC), 2015.

[19] C. Xu. Advanced flip chip and wafer level packages for 2.5D and 3D IC package technology. [PhD Thesis]. Nanyang Technological University, Singapore, 2018.

[20] C. Xu, Z. W. Zhong, and W. K. Choi. Evaluation of Fan-out Wafer Level Package strength by three-point bending testing. In 2016 IEEE 23rd International Symposium on the Physical and Failure Analysis of Integrated Circuits (IPFA), 2016. p. 297-300.

[21] J D Wu, C Y Huang, and C C Liao. Fracture Strength Characterization and Failure Analysis of Silicon Dies. Microelectronics Reliability. 2003; 43:269-277.

[22] E. Wu, I. G. Shih, Y. N. Chen, S. C. Chen, C. Z. Tsai, and C. A. Shao. Influence of grinding process on semiconductor chip strength. Presented at the 52nd Electronic Components and Technology Conference, 2002.

[23] M Y Tsai and C H Chen. Evaluation of Test Methods for Silicon Die Strength. Microelectronics Reliability. 2008; 48:933-941.

[24] Chih-Tang Peng, Chang-Ming Liu, Ji-Cheng Lin, Hsien-Chie Cheng and Kuo-Ning Chiang. Reliability Analysis and Design for the Fine-Pitch Flip Chip BGA Packaging. IEEE Transactions on Components and Packaging Technologies. 2004; 27: 684-693.

[25] H. C. Cheng, Y. H. Tsai, K. N. Chen, and J. Fang. Thermal placement optimization of multichip modules using a sequential metamodeling-based optimization approach. Applied Thermal Engineering. 2010; 30:2632-2642.

[26] C. J. Zhan, C. C. Chuang, J. Y. Juang, S. T. Lu, and T. C. Chang. Assembly and reliability characterization of 3D chip stacking with $30 \mu \mathrm{m}$ pitch lead-free solder micro bump interconnection. In Proceedings - Electronic Components and Technology Conference, 2010. p. 1043-1049.

[27] H. C. Cheng, C. F. Yu, and W. H. Chen. Strain- and strain-rate-dependent mechanical properties and behaviors of $\mathrm{Cu} 3 \mathrm{Sn}$ compound using molecular dynamics simulation. Journal of Materials Science. 2012;47: 3103-3114.

[28] Y. C. Chan, P. L. Tu, C. W. Tang, K. C. Hung, and J. K. L. Lai. Reliability studies of $\mu$ BGA solder jointseffect of Ni-Sn intermetallic compound. IEEE Transactions on Advanced Packaging. 2001;24: 25-32.

[29] M. E. Loomans, S. Vaynman, G. Ghosh, and M. E. Fine. Investigation of multi-component lead-free solders. Journal of Electronic Materials. 1994; 23:741-746.

[30] Y. Kariya and M. Otsuka. Mechanical fatigue characteristics of Sn-3.5Ag-X (X=Bi, Cu, Zn and In) solder alloys. Journal of Electronic Materials. 1998;27:1229-1235.

[31] Noboru Wade, Kepeng Wu, Johji JKunil, and Seiji Yamada. Effect of Cu, Ag and Sb on the Creep-Rupture Strength of Lead-Free Solder Alloys. Journal of Electronic Materials. 2001;30:1228-1231. 
[32] Chien-Chia Chiu, Chung-Jung Wu, Chih-Tang Peng, Kuo-Ning Chiang, Terry Ku, and Kenny Cheng. Failure Life Prediction and Factorial Design of Lead-Free Flip Chip Package. Journal of the Chinese Institute of Engineers. 2007; 30: 481-490.

[33] B Salam, N N Ekere, and R Durairaj. A Study of Inter-Metallic Compounds (IMC) Formation and Growth in Ultra-Fine Pitch Sn-Ag-Cu Lead-Free Solder Joints. Presented at the 2006 Electronic Systemintegration Technology Conference, 2006.

[34] Kenji Takahashi, Mitsuo Umemoto, Naotaka Tanaka, Kazumasa Tanida, Yoshihiko Nemoto, Yoshihiro Tomita, et al. Ultra-High-Density Interconnection Technology of Three-Dimensional Packaging. Microelectronics Reliability. 2003; 43: 1267-1279.

[35] Ye Tian, Xi Liu, Justin Chow, Yi Ping Wu, and Suresh K. Sitaraman. Comparison of IMC Growth in FlipChip Assemblies with 100- and 200- $\mu$ m-Pitch SAC305 Solder Joints. 2013 IEEE 63rd Electronic Components and Technology Conference (ECTC), 2013. p. 1005-1009.

[36] Hui-Chuan Tsai, Meng-Chieh Liao, and Chieng-Hong Lee. Formation and Growth of Intermetallics Evolution at the SAC305 and SAC0307 Solder Joints after Wave Soldering. 2010 5th International Microsystems Packaging Assembly and Circuits Technology Conference (IMPACT), 2010.p. 1-4.

[37] K S Kim, S H Huh, and K Suganuma. Effects of Intermetallic Compounds on Properties of Sn-Ag-Cu LeadFree Soldered Joints. Journal of Alloys and Compounds. 2003; 352:226-236.

[38] Xin Ma, Fengjiang Wang, Yiyu Qian, and Fusahito Yoshida. Development of Cu-Sn Intermetallic Compound at $\mathrm{Pb}$-Free Solder/Cu Joint Interface. Materials Letters. 2003;57: 3361-3365.

[39] G Y Li, X B Jiang, B Li, P Chen, and R Liao. Influence of Dopant on IMC Growth and Mechanical Properties of Sn-3.5Ag-0.7Cu Solder Joints. Presented at the 2007 International Symposium on High Density packaging and Microsystem Integration, Shanghai, 2007.

[40] Chien Wei Chang, Su Chun Yang, Chun-Te Tu, and C Robert Kao. Cross-Interaction Between Ni and Cu Across Sn Layers with Different Thickness. Journal of Electronic Materials. 2007;36:1455-1461.

(C) 2019 by the author(s). This work is licensed under a Creative Commons Attribution 4.0 International License (http://creativecommons.org/licenses/by/4.0/). Authors retain copyright of their work, with first publication rights granted to Tech Reviews Ltd. 